level of citrullination with a semi-quantitative score from $0-3$ by two blinded observers. The presence of PAD2, PAD4, alpha-enolase and fibrinogen was also determined by immunoblotting and scored. Recombinant proteins were used as positive controls and blots with secondary antibodies only were carried out to exclude non-specific cross-reactivity.

Results Citrullinated proteins were detected in 37 out of the 40 lung tissue samples, including 9 out of 10 never smokers. The number of bands and intensity was slightly increased in the COPD smokers (mean score 1.7), followed by COPD ex-smokers (1.4), smokers (1.3) and never smokers without airflow limitation (1.1). $\mathrm{PAD} 2$ was detected in all samples, with the band intensity scores correlating roughly with those seen for citrullinated proteins i.e. highest amongst the COPD smokers (mean score 1.9) and lowest amongst never smokers (mean score 1.1). PAD4 and the RA antigens alpha-enolase and fibrinogen were observed in all lung tissue in comparable amounts regardless of disease and smoking status. There was also evidence of citrullination of alpha-enolase provided by co-migration of a $\sim 50 \mathrm{KD}$ band recognised by AMC and an antienolase antibody, and the demonstration that alpha-enolase in lung tissue ran at several isoelectric spots by $2 \mathrm{D}$ electrophoresis.

Conclusions We have shown that there is widespread citrullination of proteins in lung tissue from never smokers, and that there is a modest increase with smoking and COPD. This pattern of expression corresponds to that of PAD2. There is also expression of at least two major RA autoantigens and of PAD4. This supports the hypothesis that the lung is a site for priming the ACPA response, which is enhanced by smoking and and COPD.

\section{A1.4 EARLY SIGNS OF SUBCLINICAL INFLAMMATION AND LOCAL ANTIBODY PRODUCTION IN EARLY RHEUMATOID LUNGS}

doi:10.1136/annrheumdis-2013-203214.4

${ }^{1}$ Gudrun Reynisdottir, ${ }^{2}$ Reza Karimi, ${ }^{2}$ Helga Olsen, 'Vijay Joshua, 'Aase Haj Hensvold, ${ }^{1}$ Anders Harju, 'Marianne Engström, '2Johan Grunewald, ${ }^{3}$ Sven Nyren, ${ }^{2}$ Anders Eklund, 'Lars Klareskog, ${ }^{2}$ Magnus Sköld, ${ }^{1, *}$ Anca Irinel Catrina. ${ }^{1}$ Unit of Rheumatology, Department of Medicine, Karolinska University Hospital, Karolinska Institutet, Stockholm, Sweden; ${ }^{2}$ Division of Respiratory Medicine, Department of Medicine, Karolinska Institutet, Karolinska University Hospital Solna, Stockholm, Sweden; ${ }^{3}$ Department of Molecular Medicine and Surgery, Karolinska Institutet, Karolinska University Hospital Solna, Stockholm, Sweden

Objective To investigate if lung changes are present in RA patients early in the disease process and to address the contribution of these changes to disease initiation.

Patients and Methods 21 RA patients with symptom duration less than 1 year at the time of diagnosis and naive to DMARD treatment and 8 healthy individuals were subjected to bronchoscopy and mucosal large bronchial biopsies were retrieved. Histological analysis for identification of inducible bronchia associated lymphoid tissues (iBALT), PAD enzymes, CD3, HLA-DQ and HLA-DR expression were performed. Presence of citrullinated targets were detected by immunohistochemistry using biotinylated ACPA isolated from synovial fluid of RA patients. Presence of ACPA and ACPA fine specificities were tested by ELISA in the serum and BAL of RA patients. Mass spectrometry was used for identification of citrullinated epitopes in 6 of the lung biopsies and additional 8 synovial RA biopsies.

Results iBALT formation and higher expression of CD3, HLA-DQ, HLA-DR and citrullinated targets was observed in bronchial biopsies of ACPA positive RA. A majority of serum ACPA positive RA patients subjected to lung bronchoscopy had detectable levels of ACPA in the BAL fluids both IgA and IgG. IgG from BAL fluids of ACPA-positive patients showed a higher ACPA reactivity as compared to serum IgG from the same patients. Mass spectometry identified 5 proteins in the synovium (in total 8 sites) and 4 in the lungs (in total 6 sites) containing citrullinated residues. Two vimentin derived citrullinated peptides were present in a majority of both synovial and lung biopsies with slightly higher citrullinated/unmodified peptides ratios in the smokers as compared to non-smokers.

Conclusions Lung subclinical inflammation is present already at the earliest visit to a rheumatology specialist early after disease onset in ACPA + RA patients. These findings suggest that the lungs might be the primary local initiation sites of the anti-citrulline response in RA.

\section{A1.5 EXPLORING THE ROLE OF THE LYMPH NODE MICROENVIRONMENT IN HEALTH AND DISEASE}

doi:10.1136/annrheumdis-2013-203214.5

1,2 J Hähnlein, ${ }^{1,2}$ TH Ramwadhdoebe, ${ }^{1} \mathrm{KI}$ Maijer, ${ }^{1} Y \mathrm{~K}$ Choi, ${ }^{1,2} \mathrm{NAM}$ Smits, ${ }^{3} \mathrm{M}$ Maas, ${ }^{1} \mathrm{DM}$ Gerlag, 'PP Tak, 1,2LGM van Baarsen. 'Division of Clinical Immunology \& Rheumatology, Academic Medical Center, University of Amsterdam, Amsterdam, Netherlands, ${ }^{2}$ Division of Experimental Immmunology, Academic Medical Center, University of Amsterdam, Amsterdam, Netherlands; ${ }^{3}$ Division of Radiology, Academic Medical Center, University of Amsterdam, Amsterdam, Netherlands

Background and Objective Rheumatoid arthritis (RA) is an immune-mediated inflammatory disease of unknown aetiology. Changes in the lymph nodes could precede those in the synovial joints. Recent studies reported the importance of lymph node stromal cells (LNSCs) in lymphoid homeostasis, peripheral tolerance and the adaptive immune response. Therefore, we characterised the functional capacities of LNSCs in human lymph nodes during health and different phases of RA.

Materials and Methods Individuals with arthralgia but without any evidence of arthritis upon physical examination who were positive for IgM rheumatoid factor and/or anti-citrullinated protein antibodies were included ( $\mathrm{n}=12$; RA risk group). In addition, we included patients with early arthritis (disease duration $<1$ year; $\mathrm{n}=6), \mathrm{RA}(\mathrm{n}=15)$ and healthy controls $(\mathrm{n}=8)$. All study subjects underwent ultrasound-guided inguinal lymph node biopsy. LNSCs were isolated and cultured from freshly collected lymph node needle biopsies. LNSCs originating from different culture passages were studied to investigate the effects of in vitro culture on the expression level of stromal cell associated genes, including VCAM-1, ColIVa and IL-6. In addition, we analysed the expression of Deaf1 and Aire, transcriptional regulators involved in peripheral tolerance. Functional capacities of LNSCs were studied by analysing STAT-1 and MxA mRNA induction and interleukin (IL)- 6 and IL-8 production after TLR-3 triggering by PolyI:C.

Results Cells with a fibroblast-like morphology started to grow out from the stromal part of lymph node biopsies within a few weeks of culture. Passage 0 consisted of a mixture of adherent cells, resulting in a lower expression of the measured stromal genes. From passage 1 gene expression levels for VCAM-1, ColIVa and IL-6 increased and stabilised. All LNSCs cell lines expressed Deaf1 while the expression of Aire was only detected at very low levels. LNSCs were sensitive for TLR-3 ligation by PolyI:C resulting in upregulated STAT-1 and MxA, downregulated Deaf1 and high levels of IL-6 and IL-8. Chemokines CCL19 and CCL20 were only expressed after stimulation with PolyI:C. There was a high variability between donors and interim analysis showed no clear differences between LNSCs cultured from healthy individuals, RA risk or RA patients.

Conclusions We developed a culture system for human LNSCs to facilitate research on the role of the lymph node microenvironment in the pathogenesis of RA. Cultured human LNSCs express typical stromal cell markers and are responsive for TLR-3 triggering. Interestingly, the LNSCs express the transcriptional regulator Deaf1 which may indicate peripheral tissue antigen expression by LNSCs. 